\title{
Analysis of skeletal muscle function in the C57BL6/SV129 syncoilin knockout mouse
}

\author{
Karl J. A. McCullagh • Ben Edwards · Matthew W. Kemp · Laura C. Giles • \\ Matthew Burgess · Kay E. Davies
}

Received: 21 April 2008/Accepted: 28 May 2008/Published online: 2 July 2008

(C) The Author(s) 2008

\begin{abstract}
Syncoilin is a $64-\mathrm{kDa}$ intermediate filament protein expressed in skeletal muscle and enriched at the perinucleus, sarcolemma, and myotendinous and neuromuscular junctions. Due to its pattern of cellular localization and binding partners, syncoilin is an ideal candidate to be both an important structural component of myocytes and a potential mediator of inherited myopathies. Here we present a report of a knockout mouse model for syncoilin and the results of an investigation into the effect of a syncoilin null state on striated muscle function in 6-8week-old mice. An analysis of proteins known to associate with syncoilin showed that ablation of syncoilin had no effect on absolute expression or spatial localization of desmin or alpha dystrobrevin. Our syncoilin-null animal exhibited no differences in cardiotoxin-induced muscle regeneration, voluntary wheel running, or enforced treadmill exercise capacity, relative to wild-type controls. Finally, a mechanical investigation of isolated soleus and extensor digitorum longus indicated a potential differential reduction in muscle strength and resilience. We are the first to present data identifying an increased susceptibility to
\end{abstract}

K. J. A. McCullagh, B. Edwards, and M. W. Kemp contributed equally to this work.

K. J. A. McCullagh · B. Edwards · M. W. Kemp .

L. C. Giles · M. Burgess - K. E. Davies ( $\square)$

MRC Functional Genomics Unit, Department of Physiology,

Anatomy and Genetics, University of Oxford, South Parks Road,

Oxford OX1 3QX, UK

e-mail: kay.davies@dpag.ox.ac.uk

Present Address:

K. J. A. McCullagh

Regenerative Medicine Institute (REMEDI), National Centre for Biomedical Engineering Science, National University of Ireland, Galway, Ireland muscle damage in response to an extended forced exercise regime in syncoilin-deficient muscle. This study establishes a second viable syncoilin knockout model and highlights the importance of further investigations to determine the role of syncoilin in skeletal muscle.

\section{Introduction}

Syncoilin is a recently described member of the intermediate filament (IF) family of proteins (Newey et al. 2001). So named for its enrichment at the neuromuscular synapse and its coiled-coil-forming rod domains, the syncoilin gene has been mapped to chromosome 1 in humans and to chromosome 4 in mice. The syncoilin gene comprises five coding exons that generate a $64-\mathrm{kDa}$ protein product (Newey et al. 2001). Following its discovery, subsequent yeast two-hybrid screening analysis showed that the IF protein desmin and the dystrophin-related protein $\alpha$-dystrobrevin were syncoilin binding partners in striated muscle (Newey et al. 2001; Poon et al. 2002). Histologic studies utilizing immunofluorescent and confocal microscopy have confirmed that syncoilin colocalizes under normal conditions with both desmin and $\alpha$-dystrobrevin (McCullagh et al. 2007; Newey et al. 2001; Poon et al. 2002).

One distinctive feature of syncoilin is its apparent departure from spontaneous dimer-forming behavior. Syncoilin does not appear to form a filamentous network by itself or with other IF proteins, although it may form filaments in certain myopathies (Brown et al. 2005; Poon et al. 2002).

Syncoilin is highly expressed in skeletal and cardiac muscle, and in the former it is concentrated at the sarcolemma, Z-lines, perinuclear space, myotendinous junction, and neuromuscular junction (McCullagh et al. 2007; 
Newey et al. 2001; Poon et al. 2002). Within the skeletal muscle fiber population, syncoilin is enriched at the sarcolemma and sarcoplasm of oxidative muscle fibers (McCullagh et al. 2007). Thus, the localization of syncoilin parallels that of desmin, to which it binds directly.

Desmin is the dominant IF protein in mature muscle and has been predicted to play a major role in protecting muscle from mechanical stress and is important for muscle integrity (Capetanaki et al. 2007; Costa et al. 2004). An analysis of the tensile properties of single desmin filaments has shown that it exhibits a nonlinear response to strain (notably above $50 \%$ extension) and a tensile strength of at least $240 \mathrm{mPa}$ (Kreplak et al. 2008). These properties add further weight to the theory that desmin acts as both a mediator of myocyte spatial organization and provides a physical link between adjacent contractile units.

Desmin knockout (des-/-) mice show a skeletal myopathy as well as a cardiomyopathy, and desmin aberrations have been linked to disease in humans ( $\mathrm{Li}$ et al. 1996; Milner et al. 1996; Olive et al. 2004). A study of exercise ability showed a reduction in the capacity of des-/- mice to perform both voluntary wheel running and enforced treadmill exercise. Most interestingly, the study showed that an absence of desmin was not sufficient to increase serum creatine kinase levels (a marker of sarcolemmal damage) (Haubold et al. 2003).

We have previously shown that the levels of syncoilin are reduced by $60 \%$ in these animals and that the subcellular location of syncoilin is altered. In the des-/- mice, syncoilin is lost from the Z-lines and reduced at the sarcolemma (McCullagh et al. 2007). Alterations in syncoilin expression have been reported for other myopathies, including elevated levels in dystrophic muscles of $m d x$ mice and in atrophic muscle fibers of patients with neurogenic disorders (Brown et al. 2005; Newey et al. 2001).

Although there is clear evidence showing that syncoilin binds directly to both $\alpha$-dystrobrevin and desmin, whether syncoilin has the ability to bind to these proteins simultaneously and thus form a link between the cytoskeletal IF network and the sarcolemma via the dystrophin associated protein complex (DAPC) remains to be investigated. This potential linking of the DAPC to the IF network via syncoilin is of interest since the lateral transmission of contractile force to the extracellular matrix is believed to be mediated mainly through IF proteins (Lieber et al. 2002).

The DAPC is a trans-sarcolemmal protein complex expressed in both muscle and nonmuscle tissues (Blake et al. 2002). Due to its expression patterns and its large and varied number of protein constituents, the DAPC is considered to play both a structural and a signaling role. A detailed review of the DAPC with respect to its structure and predicted role in normal skeletal muscle function and myopathy is outside of the scope of this article. The reader is referred to comprehensive reviews by Blake et al. (2002) and Davies and Nowak (2006) for further detail.

Briefly, the DAPC consists of the $\alpha$ - and $\beta$-dystroglycans and the sarcoglycans at the sarcolemma, along with the cytoplasmic components syntrophin and dystrobrevin. It is now well established that mutations in dystrophin and the sarcoglycans result in a muscular dystrophy. Aberrations in both desmin and $\alpha$-dystrobrevin have been implicated in muscular dystrophies, although in the case of $\alpha$-dystrobrevin its role in the pathophysiology of neuromuscular disease is far from clear (Jin et al. 2007; Jones et al. 2003; Paulin et al. 2004; Rees et al. 2007). The DAPC stabilizes the sarcolemma by linking the extracellular matrix (via an extracellular interaction of laminin-2 with DAPC component $\alpha$-dystroglycan) with the internal cytoskeleton and contractile apparatus of myocytes (via an interaction of dystrophin with F-actin and indirectly with the IF network via $\alpha$-dystrobrevin) (Blake et al. 2002).

In view of the findings that the loss of either desmin or dystrobrevin results in the development of muscular dystrophy in association with changes in the distribution of syncoilin, we decided to test the hypothesis that loss of syncoilin might have an adverse effect on myocyte function. We generated a syncoilin knockout mouse by standard techniques of homologous recombination in embryonic stem (ES) cells and a Cre-loxP-mediated conditional genetargeting approach. Such null mutants would allow the investigation of the role of syncoilin in skeletal and cardiac muscle. This strategy allowed us to produce two lines of mice: conditional (sync-floxed) and constitutive syncoilin (sync-/-) knockout mice. Constitutive deletion of syncoilin resulted in a viable mouse, precluding the need for tissuespecific excision of the syncoilin gene. In this article we present the results of a molecular and phenotypic characterization of striated muscle from a novel sync-/- mouse.

\section{Materials and methods}

Animals and antibodies

All mice were bred in-house and genotyped by standard PCR methods. All animal experiments were performed according to guidelines and protocols approved by the Home Office. Antibodies used were anti-syncoilin-FP 81, anti-desmin (Abcam8592), anti-desmin monoclonal antibody (DE-U-10; Sigma), anti-dystrobrevin- 1 and -2 antibody $\beta 1$ CTFP (Blake et al. 1998), anti-dysbindin antibody (Benson et al. 2001; Newey et al. 2001), anti- $\alpha$-actinin mouse (N40; Santa Cruz), anti- $\alpha$-actinin antibody (EA-53; Sigma) and anti-dystrophin antibody (Dys-2; Novacastro). A synemin antibody (antiSyn-3) that detects both $\alpha$ - and $\beta$-synemin was generated as previously described (Mizuno et al. 2004). 
Gene targeting and generation of syncoilin knockout mice

The targeting construct is shown in Fig. 1. Genomic regions of the syncoilin gene were amplified by PCR from a $129 / \mathrm{Sv}$ bacterial artificial plasmid (Wellcome Trust Sanger Institute, Cambridge, UK) which contained the syncoilin locus. The targeting construct was assembled in the pBluescript II KS+ vector. The $5^{\prime}$ arm of homology consisted of a 1.6-kb fragment upstream of syncoilin exon 2 followed by a lox $\mathrm{P}$ site and neomycin flanked by frt recombinase sites. The $3^{\prime}$ arm of homology was a 5.9-kb fragment containing exon 2 followed by a loxp site. The targeting vector was verified by sequencing and linearized with NotI before electroporation into E129/sv ES cells. Four hundred G418-resistant ES clones were selected for screening for homologous recombination by PCR and Southern blotting. Genomic DNA isolated from each clone was digested with $N c o$ I and analyzed by Southern blotting. A $5^{\prime}$ internal probe and $3^{\prime}$ external probe (indicated in Fig. 1) were generated from mouse E129 genomic DNA.

Southern blots were hybridized with radiolabeled probes and visualized by autoradiography. The $5^{\prime}$ internal probe was used to confirm recombination of the $5^{\prime}$ arm of the targeting vector, where the wild-type allele is indicated by a band at $2.3 \mathrm{~kb}$, and a $3.5-\mathrm{kb}$ band represents the correctly targeted allele. Hybridization of blots with the $3^{\prime}$ external probe was used to verify recombination of the $3^{\prime}$ arm of the targeting vector, where the wild-type allele is represented by a band at $8.2 \mathrm{~kb}$, and a $6.3-\mathrm{kb}$ band represents the correctly targeted allele.

\section{Generation and genotyping of mice}

Two independent correctly targeted ES clones were microinjected into blastocysts from C57BL/6 mice. Chimeric mice were bred with transgenic mice ubiquitously expressing Cre recombinase under the cytomegalovirus promoter (CMV), to generate germline-transmitted syncoilin heterozygous (sync+/-) mice. Sync $+/-$ mice were intercrossed to generate homozygous syncoilin null (sync-/-) mice. Offspring from intercrosses were genotyped by PCR analysis of mouse tail DNA using the following primers: forward (wtFw), 5'-ATGAAGTGATGTAAGCAGAGACTAGC-3'; reverse(wtRv), 5'-GATAGAGAATGTCCTCTAGGTTCA CAG-3'; deletion indicator primer $(\triangle \mathrm{Ex} 2 \mathrm{Rv}), 5^{\prime}$-TCCT AATCCTAACCCTACCCTAACTAA- $3^{\prime}$.

\section{RNA extraction and RT-PCR}

Total RNA was prepared from skeletal and cardiac muscle of sync $+/+$ and sync-/- mice. Dissected tissue was snapfrozen and milled in liquid nitrogen immediately before total RNA extraction using Trizol (Invitrogen) according to the manufacturer's instructions. Total RNA (300 ng) was reverse-transcribed into cDNA using random decameric primers and superscript II reverse transcriptase (Invitrogen). Generated cDNA was used as a template to perform standard PCR analysis.

Specific primers annealing to exon 2 and exon 5 (forward, 5'-AACTTCAGAACAGGAACCTGGAGGAC-3'; reverse, 5'-CACTGAAATCAGTACCAGCAGCCTAC-3') were used to amplify and test for the presence of syncoilin transcript in sync+/+ and sync-/- muscles. Standard exonic GAPDH primers were run on duplicate samples as a control for the cDNA template.

\section{Protein preparation and immunoblotting}

Skeletal or cardiac muscles were snap-frozen immediately after dissection in liquid nitrogen and stored at $-80^{\circ} \mathrm{C}$ until use. The tissue was homogenized in $300 \mu \mathrm{l}$ cold Newcastle buffer (75 mM Tris pH 6.8, 3.8\% SDS, $4 \mathrm{M}$ urea, and 20\% glycerol) with protease inhibitor cocktail (Sigma). Protein quantification was performed using the Bio-Rad Dc Protein Assay according to the manufacturer's protocol. Total protein $(40 \mu \mathrm{g})$ was separated on 8 or $10 \%$ SDS polyacrylamide gels (Bio-Rad) and electrophoretically transferred to nitrocellulose membranes. After blocking membranes overnight at $4^{\circ} \mathrm{C}$ in Tris-buffered saline containing $0.1 \%(\mathrm{v} / \mathrm{v})$ Tween20 (TBS-T) and 5\% nonfat dry milk, blots were incubated for $1 \mathrm{~h}$ with primary antibody and washed three times in TBS-T prior to incubation with horseradish peroxidase conjugated IgG in blocking buffer. Membranes were washed as previously described and developed using ECL detection reagents (Amersham) as per the manufacturer's instructions. Blots were stripped at $50^{\circ} \mathrm{C}$ for reprobing with different antibodies. Exposed film was scanned with a HP Scanjet 5400c.

\section{Single myofiber preparations}

Mouse flexor digitorum brevis muscles (FDBs) were enzymatically dissociated using an established protocol (Brown and Schneider 2002; Woods et al. 2004). The FDB was dissected free and placed into $1 \mathrm{mg} / \mathrm{ml}$ of collagenase (Type 1, Sigma) in Ringer buffer (119 mM NaCl, $5 \mathrm{mM} \mathrm{KCl}$, $1.25 \mathrm{mM} \mathrm{CaCl}_{2}, 1 \mathrm{mM} \mathrm{MgSO}, 1 \mathrm{mM} \mathrm{KH} \mathrm{PO}_{4}, 10 \mathrm{mM}$ glucose) at $37^{\circ} \mathrm{C}$ for $1.5 \mathrm{~h}$. Collagenase activity was stopped by washing the muscle with warmed Ringer's solution and the muscle was gently triturated using a Pasteur pipette until single fibers were isolated. One hundred fifty microliters of Ringer's solution containing FDB single fibers was spread across laminin-coated glass bottom culture dishes (Matek Corp., Part No P35G-1.0) and incubated in $5 \% \mathrm{CO}_{2}$ at $37^{\circ} \mathrm{C}$. After 2 h, Ringer's solution was removed and the dish was 
flooded with $4 \%$ PFA/PBS for 5 min to fix the fibers. This method yielded approximately 100-200 fibers per dish. The fibers were then permeabilized in $0.1 \%$ Triton/PBS and then processed for immunolabeling.

Primary antibodies were diluted in PBS containing $10 \%$ FCS and applied to the fiber preparations. After an overnight incubation at $4^{\circ} \mathrm{C}$, fiber preparations were rinsed in PBS followed by incubation with secondary antibodies (Molecular Probes); Alexa fluor 594 and/or Alexa fluor 488 conjugated IgG diluted (1:2000) in PBS, 10\% FCS for $2 \mathrm{~h}$ at room temperature. Finally, fiber preparations were washed thoroughly in PBS and mounted in an aqueous medium (VectorShield) with DAPI. Finished preparations were viewed under a Zeiss fluorescent photomicroscope or Zeiss 410 confocal laser-scanning microscope. Digital images were extracted using Axiovision LE 4.3 software.

\section{Measurement of contractile properties}

Mechanical measurements of skeletal muscle performance in vitro were performed using a previously described protocol (with minor adaptations) (Moens et al. 1993). Briefly, extensor digitorum longus (EDL) and soleus muscles were dissected from 8-week-old male mice killed by cervical dislocation. The muscles were pinned out in oxygenated Ringer's solution at room temperature to a length approximating that seen in situ. Oxygenated $\left(95 \% \mathrm{O}_{2} / 5 \% \mathrm{CO}_{2}\right)$ mouse Ringer's solution ( $\mathrm{pH} 7.4$ ) was made up to include $118 \mathrm{mM} \mathrm{NaCl}, 24.8 \mathrm{mM} \mathrm{NaHCO} 3,4.75 \mathrm{mM} \mathrm{KCl}$, $2.54 \mathrm{mM} \mathrm{CaCl}_{2}, 11.8 \mathrm{mM} \mathrm{MgSO}_{4}, 11.8 \mathrm{mM} \mathrm{KH}_{2} \mathrm{PO}_{4}$, and $10 \mathrm{mM}$ glucose. Muscles were experimented on within $4 \mathrm{~h}$ of excision. The freshly dissected muscles were mounted onto the muscle lever of the Intact Muscle Measurement System (Aurora Scientific Inc.) using 5-0 silk suture, and maintained throughout the protocol at room temperature in oxygenated Ringer's solution. The muscles were stimulated using platinum electrodes connected to a Aurora 701B stimulator. The optimum length was identified, and then the maximum isometric tetanic force was measured (EDL, $120 \mathrm{~Hz}, 500 \mathrm{~ms}$; soleus, $80 \mathrm{~Hz}, 800 \mathrm{~ms}$ ). Force measurements were normalized to muscle cross-sectional area. The wet weight of the muscles was recorded at the end of the experiment. Data were captured and analyzed using the DMC/DMA Dynamic Muscle Control and Analysis software (Aurora Scientific Inc.).

\section{Cardiotoxin-induced damage of hind limb muscle}

Sex-matched adult mice were induced and maintained under isofluorane inhalation anesthetic as discussed above. A small incision was made to expose the tibialis anterior. Using a $100-\mu \mathrm{l}$ insulin syringe, $50 \mu \mathrm{l}$ of a $10 \mu \mathrm{M}$ solution of Cardiotoxin 1 (Sigma Aldrich, Dorset, UK) dissolved in sterile
Fig. 1 Targeting of the syncoilin gene. (a) The top of the diagram shows the targeting DNA vector with relevant restriction sites, constructed by subcloning genomic syncoilin fragments, a pgk-Neopositive selection cassette, and a CMV-DTA-negative selection cassette into a modified pBluescript vector. The genomic syncoilin fragments (short $5^{\prime}$ arm and long $3^{\prime}$ arm) were PCR amplified from a bacterial artificial chromosome. The long "arm" was modified by insertion of a $\operatorname{lox} \mathrm{P}$ site and $N c o \mathrm{I}$ restriction site after exon 2. Filled boxes depict exons, loxP sites are shown as filled arrows, and frt in open boxes signify $f p$ recombinase sites. The second diagram is the relevant genomic locus of syncoilin. Probes used for Southern blotting analysis of homologous recombination events are shown as black bars below intronic and exon regions; ext, external probe; int, internal probe; and ex 2 , exon 2 probe. Primers used to genotype mice are shown as horizontal arrows. The third diagram represents the conditional (floxed) allele after homologous recombination in ES cells. The bottom diagram shows the allele after in vivo Cre-loxPmediated deletion of exon 2. PGK-Neo, neomyocin gene under the phosphoglycerine kinase promoter; CMV-DTA, diphtheria toxin A gene under the cytomegalovirus promoter. (b) Southern blot of NcoIdigested genomic DNA from two correctly targeted ES clones (+/-) and a wild-type $(+/+)$ clone. The upper panel shows the autoradiography after hybridization with a $3^{\prime}$ external probe, shown in (a). The 8.2-kb and 6.28-kb bands represent the expected wild-type and targeted alleles, respectively. The lower panel shows the same clones reprobed with a $5^{\prime}$ internal probe and expected bands $2.3 \mathrm{~kb}$ and $3.5 \mathrm{~kb}$ were detected for wild-type and targeted alleles, respectively. (c) Representative PCR genotype for wild-type $(+/+)$, heterozygous $(+/-)$, and homozygous $(-/-)$ mice using a triple primer set shown in panel A. (d) RT-PCR analysis of RNA extracted from tibialis anterior muscle (TA), soleus (Sol), and heart (Hrt) using primers from exons 2 and 5 of the syncoilin gene (see Methods). A 500-bp fragment was amplified from sync+/+ but not from sync-/- mouse RNA. GAPDH primers were included as a control for the cDNA template

PBS was introduced into the muscle belly. The overlying skin was sutured to close the wound. The contralateral limb was treated with an identical volume of PBS to serve as a control. Three mice of each genotype were treated for each timepoint. Following treatment, mice were aged for 1, 3, 5, 7, or 21 days. Treated and control tibialis anterior muscle for histologic analysis was dissected, embedded in OCT, and snap-frozen in liquid nitrogen-cooled isopentane. Treated and control tibialis anterior muscle for RNA analysis was dissected and snap-frozen in liquid nitrogen.

\section{Morphologic analysis of myoregeneration following cardiotoxin treatment}

OCT-embedded cardiotoxin-treated muscle and PBS-treated tibialis anterior muscle were sectioned on a cryostat to a thickness of 9-12 $\mu \mathrm{m}$. Sections were air-dried at room temperature, rapidly fixed in absolute ethanol, then stained with hemotoxylin and eosin and mounted in an aqueous mounting media using standard histologic techniques.

Analysis of myoregeneration marker induction

Total RNA extraction and generation of cDNA from excised cardiotoxin- and PBS-treated tibialis anterior 


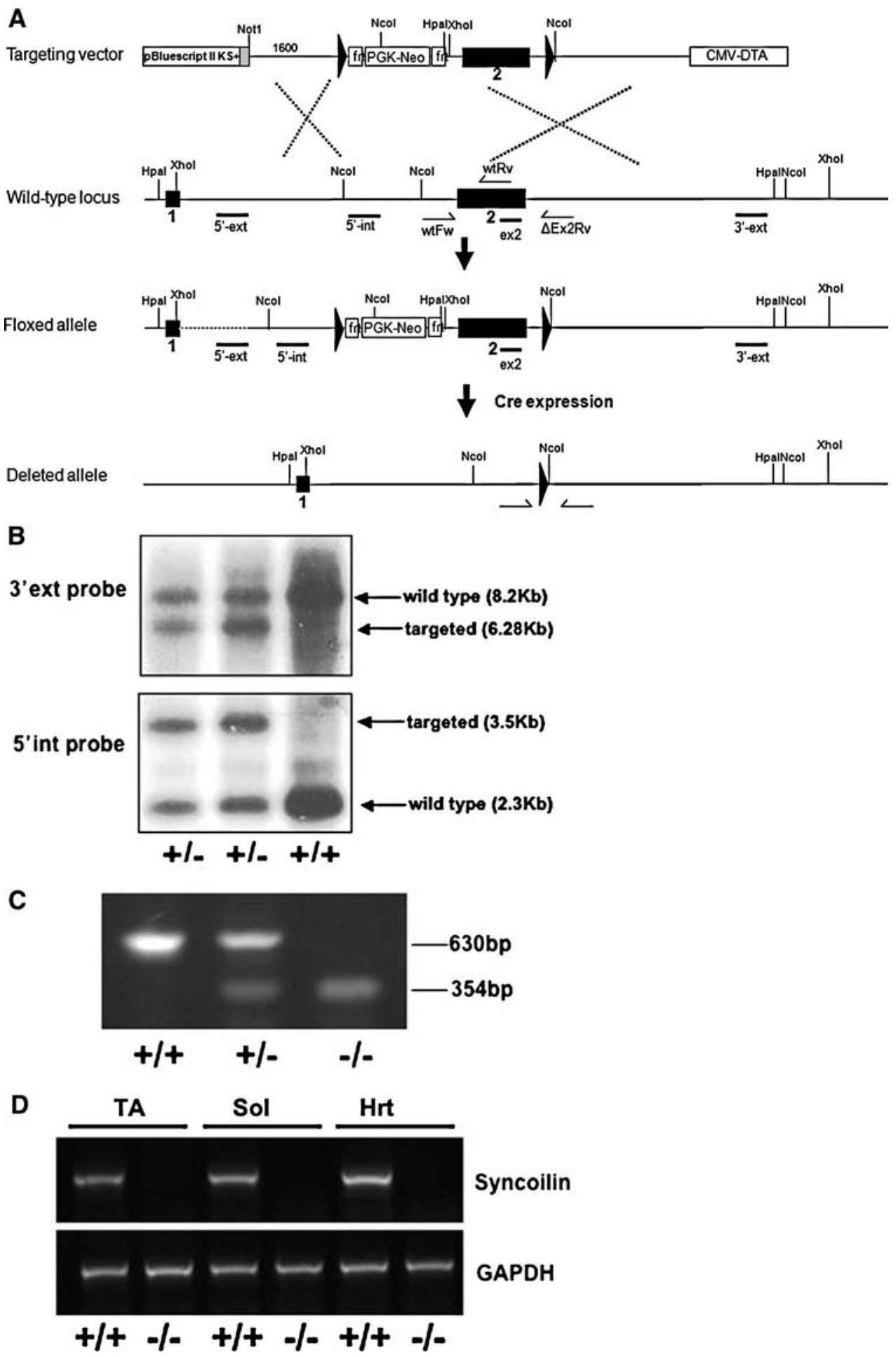

tissue were performed as described above. Semiquantitative PCR analysis of the cell cycle-dependent kinase inhibitors p21CIP1, p27KIP1, p57KIP2 and myogenin was performed as described previously (Koo et al. 2007).
Measurement of voluntary wheel running activity

To investigate the effect of syncoilin ablation on activity levels, we monitored voluntary wheel running in two groups $(n=6)$ of 6-week-old male sync $+/+$ and sync-/- 
mice. Mice were placed in individual cages supplemented with a monitored running wheel. Following an acclimatization period of 1 week, the total number of wheel revolutions performed by each animal was electronically recorded for an additional 7-day period using the ClockLab software suite (Actimetrics Software).

Measurement of enforced treadmill exercise capacity and resultant muscle damage

Treadmill exercise is a well-established means of analyzing muscle function in rodents (Armand et al. 2003). Two groups of 6-week-old male sync+/+ and sync-/- mice were exercised on a motorized treadmill (Exer6-M, Columbus Instruments). The regimen consisted of 5 days of familiarization running ( $10 \mathrm{~m} / \mathrm{min}$ for $15 \mathrm{~min}), 9$ days of endurance running ( $17 \mathrm{~m} / \mathrm{min}$ for $45 \mathrm{~min})$, and 1 day of exhaustion running $(17 \mathrm{~m} / \mathrm{min}$ for $45 \mathrm{~min}$ followed by an increase in speed of $1 \mathrm{~m} / \mathrm{min}$ for $15 \mathrm{~min}$ ). Each of the endurance/exhaustion running stages began with a 5-min warm-up period at a speed of $10 \mathrm{~m} / \mathrm{min}$. Animals were rested for 2 days after each 5-day block of running. The weight of each animal was recorded before and after each running session.

Animals were killed immediately following the final running session and approximately $100 \mu \mathrm{l}$ of venous blood was collected from the exposed jugular vein. Following a 3-h incubation on wet ice, serum was isolated by centrifugation in a benchtop centrifuge for $5 \mathrm{~min}$ at 14,000 rpm. Serum creatine kinase (CK) analysis was performed using a kinetic UV serum analysis according to manufacturer's instructions (OSR6179, Olympus R\&D, Ireland). Muscle was dissected for hemotoxylin and eosin staining as described previously. Evans Blue staining of isolated muscle was performed in $1 \mathrm{ml}$ of mouse Ringer's solution supplemented with $0.1 \%$ (w/v) Evans Blue dye. Muscle was incubated in solution for $2.5 \mathrm{~h}$ at $37^{\circ} \mathrm{C}$ before being washed in mouse Ringer's solution and embedded in OCT as described previously.

\section{Results}

Generation of gene-targeted syncoilin-null mice

We generated homozygous syncoilin knockout (sync-/-) mice by deleting the gene by in vivo Cre-loxP-mediated excision of exon 2 , which encodes $85 \%$ of the amino acid sequence (Fig. 1a). This strategy was predicted to ensure the ablation of functional syncoilin expression in knockout animals. The targeting vector was electroporated into E129/ $s v$ embryonic stem (ES) cells, and 167 clones were screened. Twenty-four clones were identified that had undergone homologous recombination (example shown in Fig. 1a). Two independent clones were each injected into blastocysts from C57BL/6 mice and then reimplanted to generate chimeric mice, bearing the floxed targeted syncoilin allele. Chimeric mice were bred with female CMV-Cre-expressing transgenic mice to excise exon 2 of syncoilin in vivo and give rise to germline-transmitted heterozygous $(s y n c-/+)$ mice. In vivo mediated excision of exon 2 was verified by PCR genotyping. Heterozygous mice were interbred to generate homozygous syncoilin knockout (sync-/-) mice, which were confirmed by PCR genotyping (Fig. 1c) and RT-PCR analysis for exons 2-5 of RNA from tibialis anterior, soleus, and heart muscles (Fig. 1d). Different genotypes (sync $+/+$, sync $+/-$, and sync -/-) were obtained in the expected Mendelian ratio. Syncoilin-null mice were viable and were indistinguishable from their wild-type littermates in appearance, behavior, and body weight.

Syncoilin protein analysis in sync-/- mice

Western blotting analysis of muscle protein from sync-/mice with a polyclonal-antibody recognizing full-length (FL) syncoilin protein confirmed that we had ablated syncoilin expression (Fig. 2). Immunoblotting of the above muscle samples with an antibody recognizing an amino terminal $(\mathrm{N})$ epitope (amino acids 1-146; encoded by exons 1 and 2), which includes the translation initiation site, gave the same results as the full-length syncoilin antibody, indicating that no aberrant syncoilin protein could be detected in sync-/- tissues.

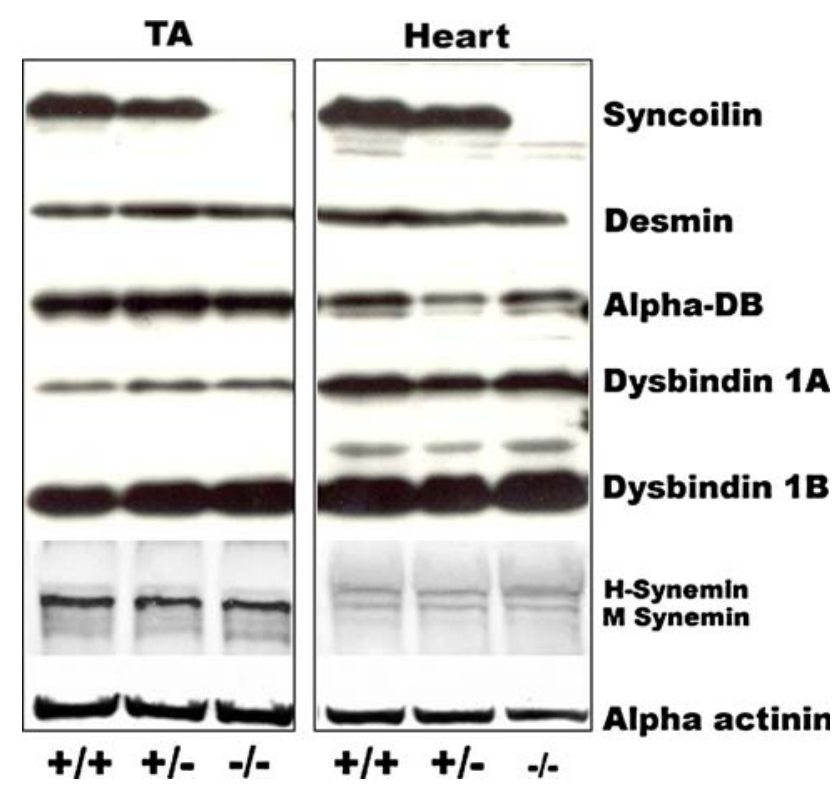

Fig. 2 Western blot analysis of syncoilin and associated muscle proteins. Images show altered syncoilin expression in $+/+,+/-$, and -/- animals, without a resultant change in syncoilin-associated proteins 
Analysis of syncoilin-binding proteins and other muscle proteins in $s y n c-/-$ mice

DAPC-associated and syncoilin-binding partner proteins were quantified by Western blotting from total skeletal and cardiac muscle protein prepared from sync $+/+$, sync $+/-$, and $s y n c-/-$ mice. Binding partners of syncoilin, desmin and $\alpha$-dystrobrevin were unaffected in sync $+/+$ and sync-/- mouse skeletal or cardiac muscle (Fig. 2). An antibody recognizing synemin isoforms $\mathrm{H}$ and $\mathrm{M}$ revealed no variation in muscles from the different syncoilin mouse genotypes (Fig. 2). Equal protein loading was demonstrated by similar $\alpha$-actinin levels between samples.

To assess if the absence of syncoilin affects the subcellular localization of syncoilin-binding proteins, the sarcomeric Z-lines, or other cytoskeletal proteins, we performed immunofluorescence on isolated myofibers. Fibers immunostained for desmin revealed no change in their normal location, with fibers from both $s y n c+/+$ and sync-/- mice showing normal localization to Z-lines and myotendinous junctions (ends of myofibers) (Fig. 3). $\alpha$-Dystrobrevin immunostaining (data not shown) also exhibited a normal distribution in both $s y n c+/+$ and sync-/- fiber preparations. Thus, the subcellular localization of known syncoilin-binding partners is not dependent on the presence of syncoilin in muscle. Immunostaining of fibers for the Z-line protein $\alpha$-actinin gave the expected Z-disc striated pattern staining in both $s y n c+/+$ and sync-/- myofibers (Fig. 3).
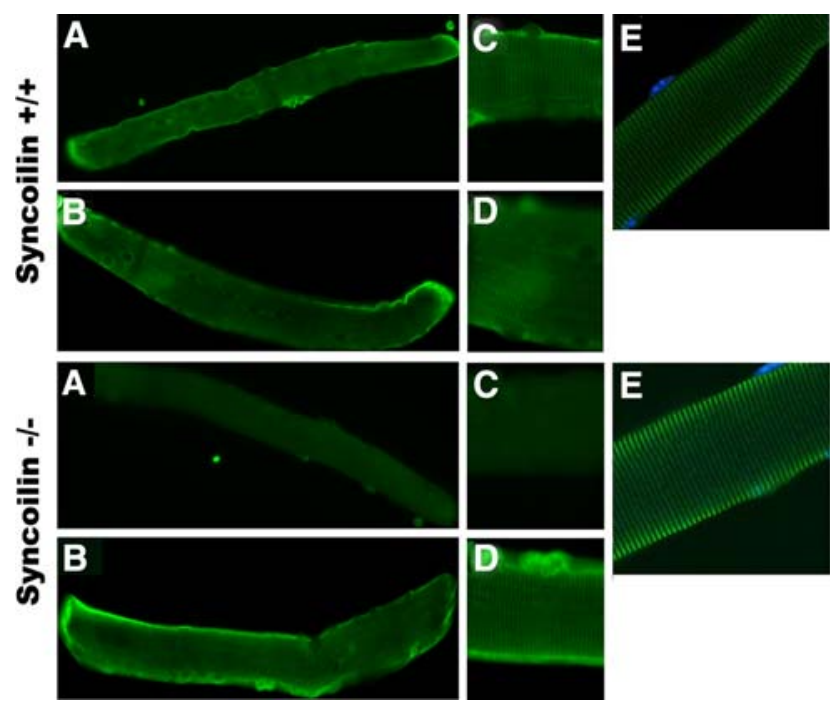

Fig. 3 Immunostaining of single-fiber preparations from flexor digitalis brevis in sync $+/+$ and sync-/ - mice. Localization of syncoilin to the Z-lines, sarcolemma, and MTJ is evident in the sync $+/+$ animal and lost in the sync-/- animal. Desmin and $\alpha$-actinin organization shows no evidence of disruption as a result of syncoilin ablation. (a) Syncoilin staining across whole fiber. (b) Desmin staining across whole fiber. (c) Syncoilin staining at Zlines. (d) Desmin staining at Z-lines. (e) $\alpha$-Actinin staining at $Z$-lines
Morphology of skeletal and cardiac muscle in sync-/mice

We examined the morphology of the more highly active muscles in 8-week-old mice by light microscopy of hematoxylin and eosin-stained sections of soleus, diaphragm, and heart from sync $+/+$ and sync-/- mice (Fig. 4). Histologic analysis revealed no gross differences between genotypes, and there was no sign of any dystrophic features or abnormal morphology in skeletal and cardiac muscle from $s y n c-/-$ mice.

Absence of syncoilin reduces contractile function in skeletal muscles

To investigate the effect of syncoilin ablation on maximum isometric force generation, we measured force production in isolated soleus and extensor digitorum (EDL) muscles from 8 -week-old sync+/+ and sync-/- mice. Four EDL muscles were assayed for each genotype. For the soleus analysis, eight sync+/+ and six sync-/- muscles were assayed. Isometric contractile testing of EDL muscles from sync-/- mice revealed a strongly significant decrease in normalized maximum force generation of $-27.9 \%$ compared with sync $+/+$ mice $\left(25.86 \mathrm{~N} / \mathrm{cm}^{2}\right.$ vs. $35.89 \mathrm{~N} / \mathrm{cm}^{2}$; $p<0.05$ ) (Fig. 6a), whereas there was no significant difference observed between the soleus muscles of $s y n c+/+$ and sync-/- mice (Fig. 5).

Ablation of syncoilin does not elicit a morphologic change in the rate of myoregeneration

To investigate any changes in muscle regeneration due to the absence of syncoilin expression, we assessed regeneration at five time points, 1, 3, 5, 7, and 21 days, following the intramuscular administration of cardiotoxin to the tibialis anterior muscle in sync $+/+$ and sync-/- mice. Figure 6a shows representative images of muscle regeneration from the $s y n c+/+$ and $s y n c-/-$ groups $(n=3$ for each genotype) across the five time points. No obvious differences in degeneration, infiltration, or regeneration (including the number of centralized nuclei at 21 days) were observed between sync $+/+$ and $s y n c-/-$ animals.

Ablation of syncoilin does not elicit an alteration in markers of myogenic differentiation

To investigate any potential difference in the kinetics of skeletal myocyte regeneration between sync $+/+$ and sync-/- animals, we performed a semiquantitative assessment of the relative RNA levels of the cell cycledependent kinase inhibitors p21CIP1, p27KIP1, p57KIP2 and myogenin in cardiotoxin-treated tibialis anterior muscle 
Fig. 4 Hematoxylin and eosin staining of highly solicited muscles, soleus, diaphragm, and heart, from 8-week-old sync+/ + and sync-/- mice. No morphologic differences were noticed in any muscle types

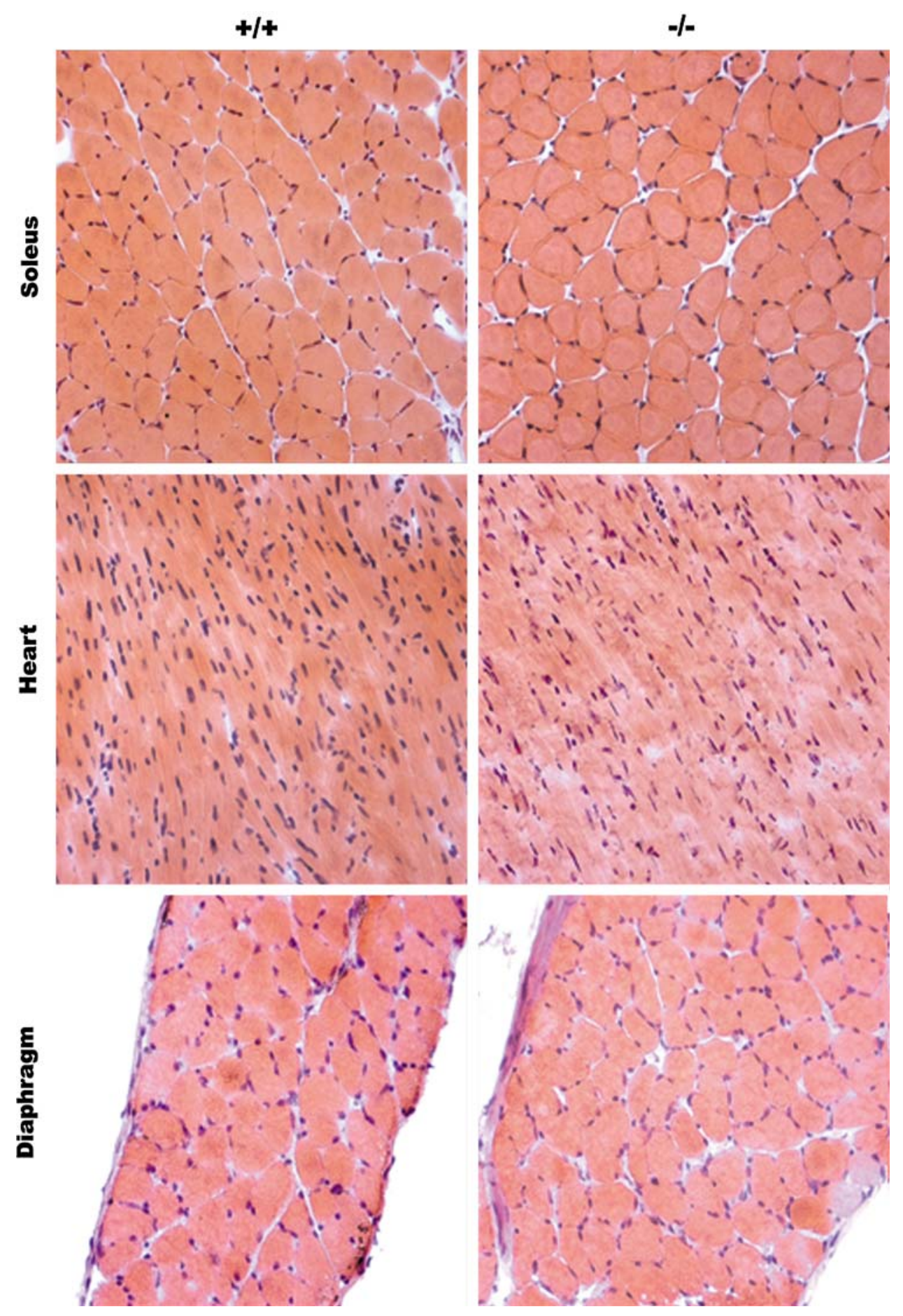

at $1,3,5$, and 7 days after administration. An increase in cell cycle-dependent kinase inhibitors can be correlated with the initiation of myogenic differentiation at the expense of cell cycling. As such, these transcript levels can be used as markers of myoregeneration kinetics (Koo et al. 2007). No obvious difference in transcript levels was detected between the synct/+ and sync-/- animals, indicating that the regeneration kinetics of skeletal muscle regeneration are not altered by the ablation of syncoilin (Fig. 6b).
Ablation of syncoilin does not produce a statistically significant difference in voluntary wheel running

A strong interanimal variation in running levels was identified in both groups $(s y n c+/+$ and sync-/-) of ageand sex-matched mice. Total individual (A) and group average (B) revolution counts are shown in Fig. 7 Individual cage positions are presented in paired form to compensate for any potential differential effect in 
Fig. 5 Contractile parameters of muscles from 8-week-old mice. Maximum isometric force of EDL and soleus muscles from sync $+/+$ and sync-/mice. EDL muscles from sync-/- mice generated $27.9 \%$ less force $\left(25.86 \mathrm{~N} / \mathrm{cm}^{2}\right)$ than from sync $+/+$ mice $(35.89 \mathrm{~N} /$ $\left.\mathrm{cm}^{2}\right) . * p<0.05$
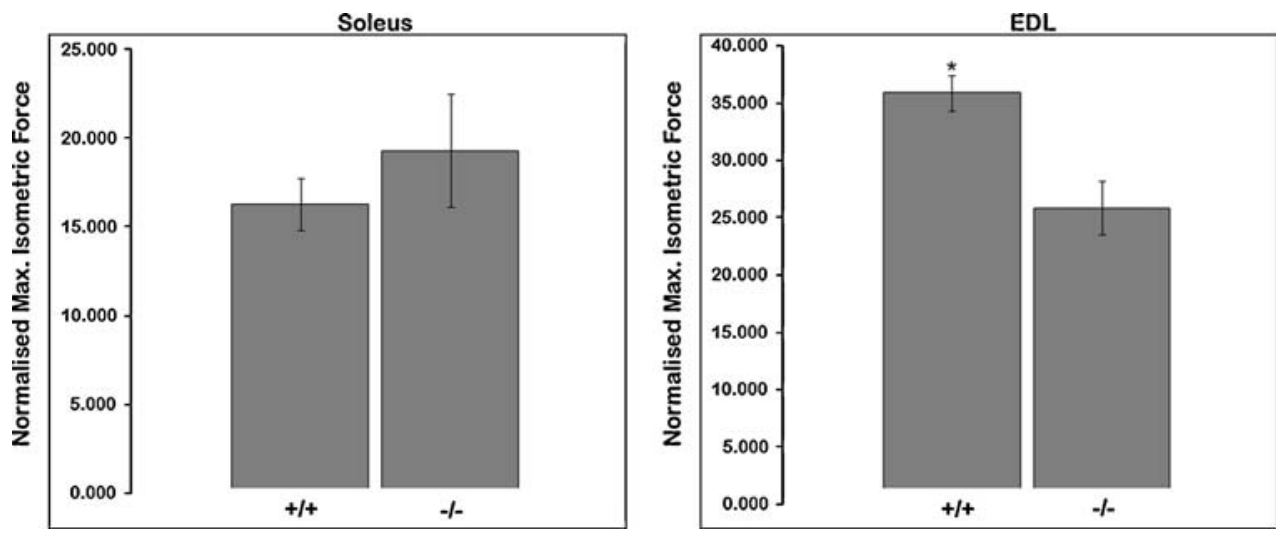

environment. Two-tailed analysis using Student's $t$ test did not identify a statistically significant difference between the averaged activity scores $(p=0.57)$ of the $s y n c+/+$ and sync-/- groups.

Ablation of syncoilin induces increased muscle damage without producing an observable difference in enforced treadmill running ability

An analysis of the enforced exercise capacity of $s y n c+/+$ and sync-l- mice failed to demonstrate a reduction in the ability of the sync-/- group to complete the prescribed exercise protocols. Eighty-three percent of $s y n c+/+$ and $100 \%$ of sync-/- mice successfully completed the final 60-min exhaustion test, reaching a maximum speed of $32 \mathrm{~m} / \mathrm{min}$ after $60 \mathrm{~min}$ of running. All animals continued to gain weight normally over the course of the study (data not shown). Figure 8 shows the results of a histologic examination of muscle morphology following 15 running sessions. No obvious differences in the gross morphology of sync $+/+$ and $s y n c-/-$ transverse sections were detected. A very small number of centralized nuclei were detected in both sync $+/+$ and sync-/- tibialis anterior sections. Evans Blue staining of isolated muscle did not indicate increased dye penetration (and therefore sarcolemmal damage) in sync-/- muscle.

$\mathrm{CK}$ analysis of serum taken from sync+/+ and sync-/animals ( $n=6$ per genotype) immediately following the completion of their running protocol showed a significant ( $p<0.05$, two-tailed Student's $t$ test) increase in serum CK levels in the sync-/- population (Table 1).

\section{Discussion}

Syncoilin is a $64-\mathrm{kDa}$ intermediate filament-like protein expressed in skeletal and cardiac muscle that is found to be enriched at the perinucleus, the Z-lines, and at the sarcolemma and neuromuscular junction (Newey et al. 2001).
Previous studies have shown that syncoilin interacts with both $\alpha$-dystrobrevin (a DAPC component) and desmin (the major intermediate filament expressed in mature myocytes) (Newey et al. 2001; Poon et al. 2002). By ablating in excess of $80 \%$ of the syncoilin-coding sequence, we have generated a mouse model that is null $(-/-)$ for syncoilin expression.

Developmentally, sync $+/+$ and $s y n c-/-$ mice are indistinguishable. Protein expression analysis of the syncoilin-binding partners desmin and $\alpha$-dystrobrevin failed to demonstrate a difference in their net concentrations in muscle derived from $s y n c+/+$ and $s y n c-/-$ mice. Immunofluorescent staining of single-fiber preparations of flexor digitalis brevis muscle demonstrated no difference in desmin or $\alpha$-dystrobrevin localization between sync $+/+$ and sync-/- mice. Together, these data indicate that syncoilin is not essential for either the correct cellular localization or expression of these proteins (Figs. 2 and 3).

The treatment of skeletal muscle with myonecrotic cardiotoxin induces widespread necrosis followed by a well characterized cascade of satellite cell activation, proliferation, differentiation, and maturation (Shi and Garry 2006). Our histologic analysis of cardiotoxin-treated tibialis anterior muscle yielded no detectable difference in regenerating muscle at any of the time points between sync $+/+$ and sync-/- mice. Semiquantitative PCR analysis of myogenin, p21, p27, and p57 expression in cardiotoxintreated tissue also failed to elicit a noticeable difference in transcript levels. When their expression is upregulated, these proteins act to initiate myogenic differentiation and prevent the cell from re-entering the cell replication cycle (Koo et al. 2007). These findings indicate that a lack of syncoilin does not obviously retard muscle tissue regeneration and that thus syncoilin is unlikely to play an essential role in this process. The results of both our voluntary running and enforced treadmill exercise studies indicate that phenotypically syncoilin is dispensable for the normal mechanical function of striated mucle under exercise conditions. 
Fig. 6 (a) Regeneration of tibialis anterior muscle following treatment with cardiotoxin. Representative images $(n=3$ for each genotype at each time point) of hemotoxylin and eosin staining of 9-12- $\mu \mathrm{m}$ cryosections taken from animals at $1,3,5,7$, and 21 days after toxin administration. No obvious difference in degeneration, basophilic infiltration, or regeneration was noticed between sync $+/+$ and sync-/mice. (b) Myogenic

differentiation analysis.

Semiquantitative PCR analysis of p21CIP1, p27KIP1, p57KIP2, and myogenin RNA transcript levels in tibialis anterior at 1,3 , 5 , and 7 days following cardiotoxin administration. Images (which are representative of $n=3$ for each genotype at each time point) indicate no difference in regeneration kinetics between sync $+/+$ and sync-/- animals
A

CTOX

1 Day
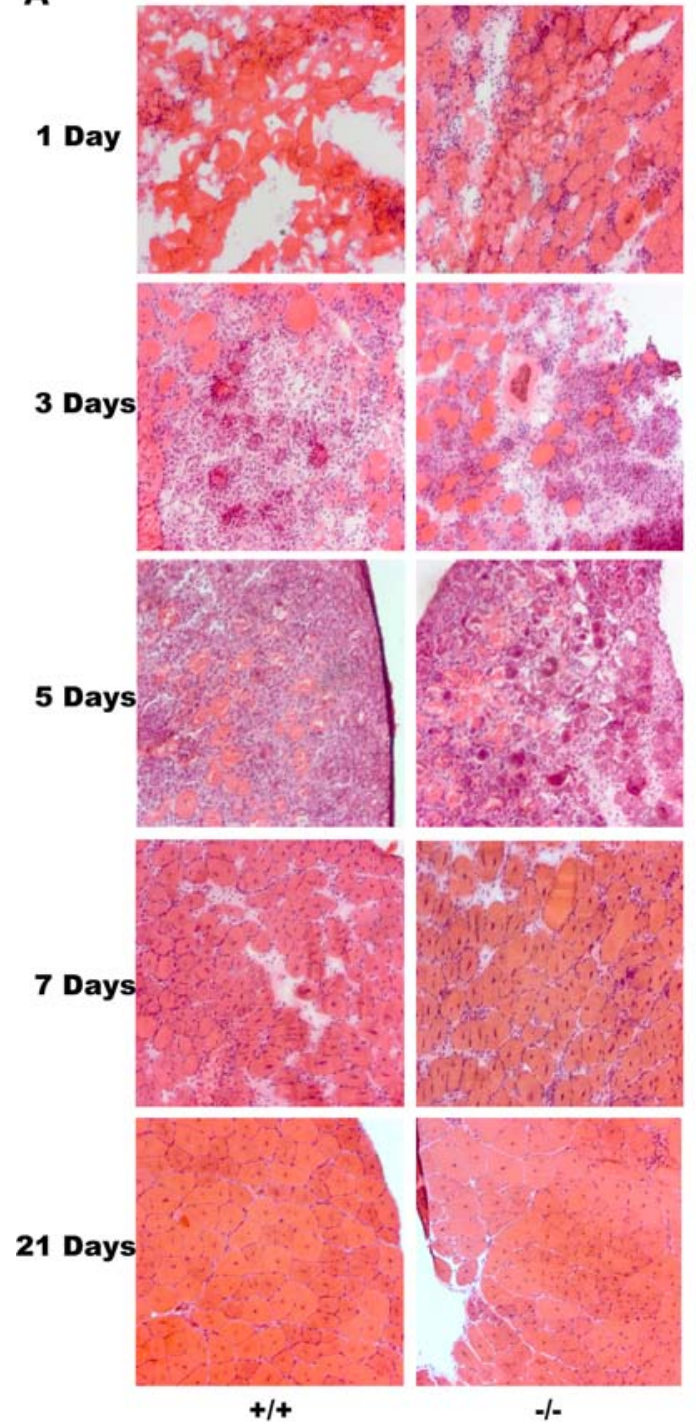

$-/-$

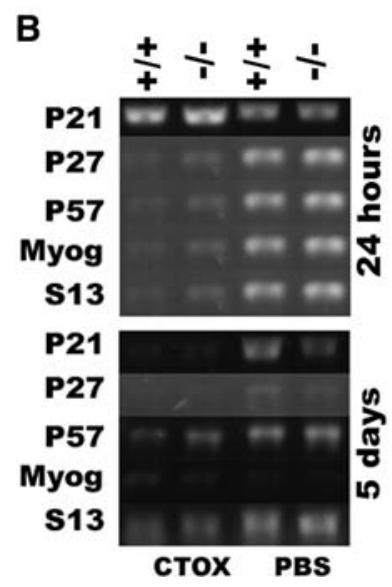

B
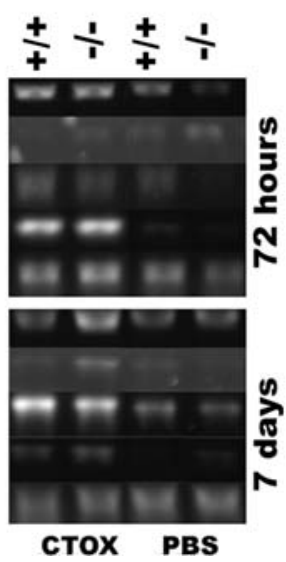

Interestingly, an investigation of contractile function in isolated soleus and EDL demonstrated a reduction of nearly $30 \%$ in the maximum force generated by sync-/EDL compared with sync+// EDL (although no difference in the soleus analysis) (Fig. 5). EDL comprises mostly fast (type II) myofibers, in contrast to soleus muscle which is populated mainly by slow (type I) myofibers. The reduction in maximum force generation seen in EDL may indicate 

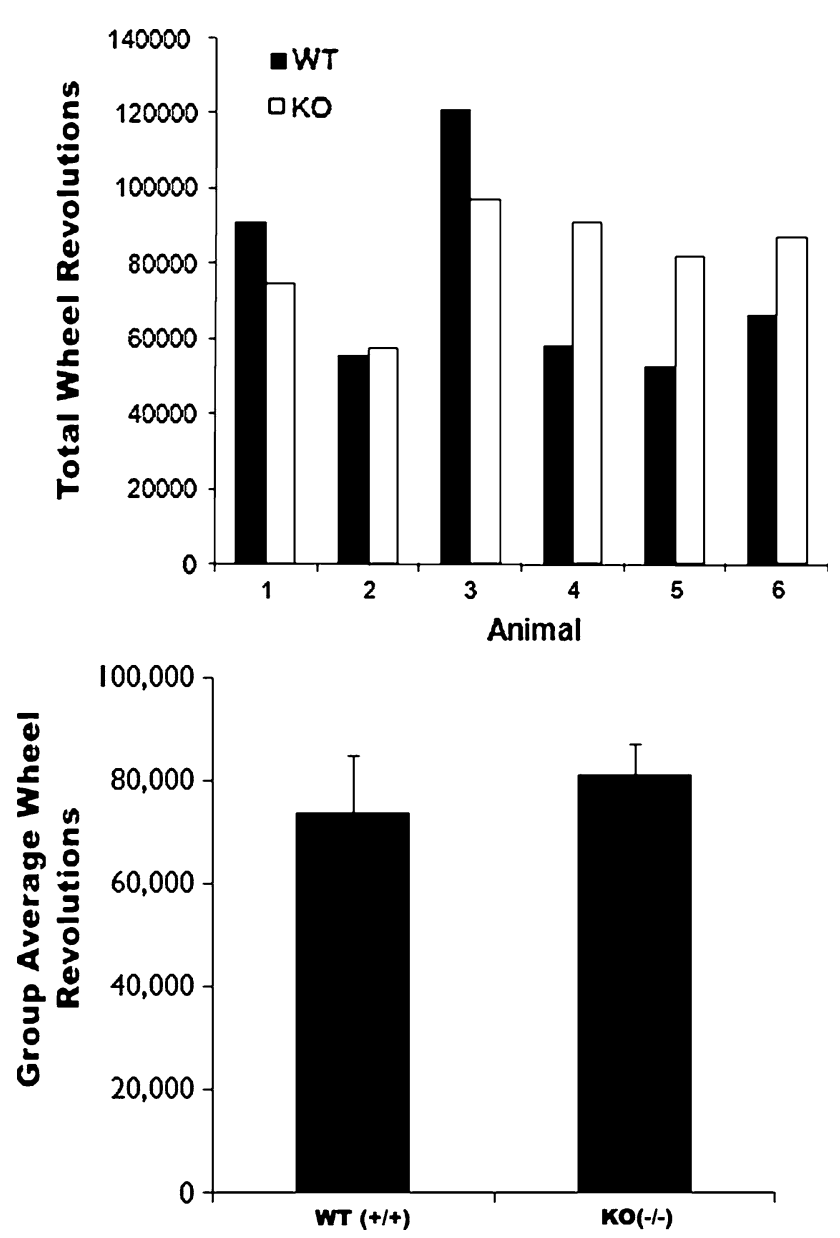

Fig. 7 Seven-day analysis of voluntary wheel running in $s y n c+/+$ and $s y n c-/-$ mice. No significant difference was detected in the total activity levels of the two groups

that syncoilin plays varying roles or has a variable functional redundancy in different fiber types. The identification of a force reduction in sync-/- EDL is in accordance with a similar analysis performed by Zhang et al. (2008). Using an alternative syncoilin-null model (generated by the introduction of a point mutation resulting in the aberrant mRNA splicing of the second syncoilin exon), this study reinforces the findings presented in our own analysis, namely, that syncoilin appears to be largely redundant for striated muscle development and regeneration (Zhang et al. 2008).

Our analysis of serum CK levels following 3 weeks of enforced treadmill exercise is the first report of a functionally induced myopathy deriving from an absence of syncoilin (Table 1). Elevated serum CK levels are widely used as a marker of sarcolemmal damage. This finding is in contrast to earlier studies performed on des-/- mice, which failed to exhibit an increase in serum CK levels after a bout of enforced treadmill exercise (Haulbold et al. 2003).
An analysis of serum CK levels in des-/- mice was performed immediately following and $24 \mathrm{~h}$ after a brief period of low-speed downhill running as described by Vilquin et al. (1998) for use in the dystrophin $m d x$ mouse model (Haubold et al. 2003; Vilquin et al. 1998). The results of this study did not show a difference in CK levels between wild-type and des-/- animals either immediately after or 1 day after exercise.

The $m d x$ mouse is used as a model of dystrophin ablation-induced muscular dystrophy and exhibits a severe myopathic phenotype (Vilquin et al. 1998). The phenotype of a des-/- mouse is far milder than that exhibited by the $m d x$ mouse, although des-/- mice still exhibit a reduction in running ability when compared with wild-type animals (Haubold et al. 2003). At a molecular level, a loss of desmin does not appear to induce disruption of the essential DAPC as is seen with a loss of dystrophin. Although it is surprising that a loss of syncoilin induces an increase in serum CK levels whereas a loss of desmin does not (given the proposed role of syncoilin and the abundance of desmin in mature myocytes), it is a possibility that the exercise regimen applied to the des-/- mice was not sufficient in either duration or intensity to induce sufficient damage to cause mechanical stress.

The precise function of syncoilin remains undelineated. However, we do possess several pieces of evidence that can assist us in generating hypotheses about the role(s) of syncoilin in muscle. Given that syncoilin is a member of the IF protein family (at least at a sequence homology level), we can reasonably expect that it plays a role in line with that of other IF proteins, which, among others, involves protecting cells from mechanical stress (Parry et al. 2007). Syncoilin's association with the DAPC and other IF proteins (in particular, the finding that it binds to desmin) adds weight to it having a structural role in muscle. Our studies indicate that at least in an acute setting there are sufficient mechanisms in existence in myocytes to cope with a loss of syncoilin. One such potential compensatory mechanism may exist in skeletal muscle in the form of the type IV IF protein synemin, although it is worth noting that there are significant size and structural differences between these two IF proteins.

Synemin is a large IF protein expressed in all types of human muscle cells as two splice variants, $\alpha$-synemin and $\beta$-synemin (Sun et al. 2008). Synemin has been shown to act as a binding partner for a range of cytoskeletal and structural proteins and contains a binding site for $\alpha$-actinin (Bellin et al. 1999, 2001). Recent studies have indicated that the two variants may play different roles in muscle, with the ability to interact with vinculin and metavinculin limited to $\alpha$-synemin, which contains an additional C-terminal 312-residue insert not found in $\beta$-synemin (Sun et al. 2008). Importantly for our investigation of syncoilin, 
Fig. 8 Histologic analysis of tibialis anterior (TA), soleus (SOL), and extensor digitorum longus (EDL) showing no overt difference between $s y n c+/+$ and sync-/- mice (as assessed by hemotoxylin and eosin staining and Evans Blue staining). Images are representative of sections taken from three animals per genotype
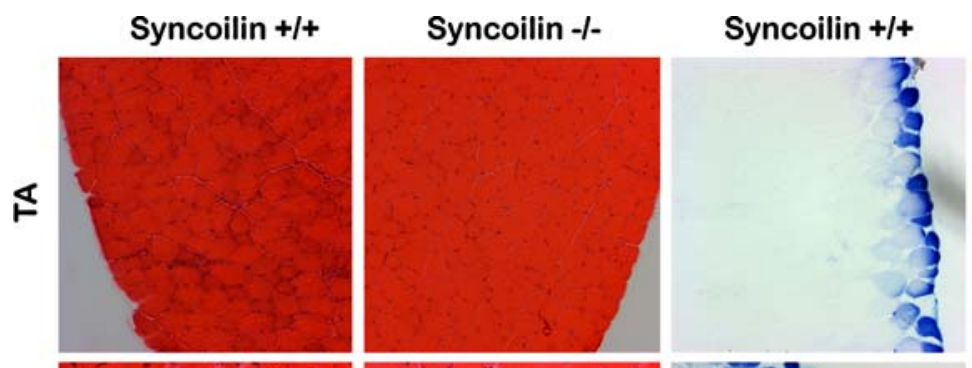

Syncoilin -1-
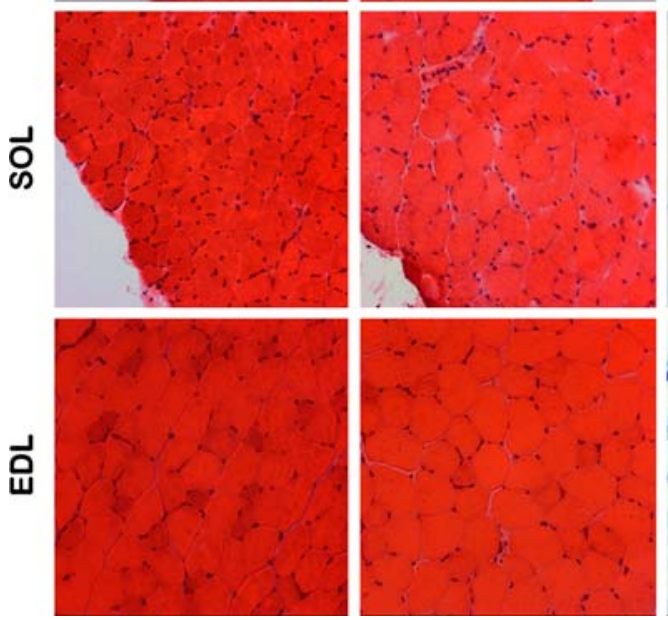
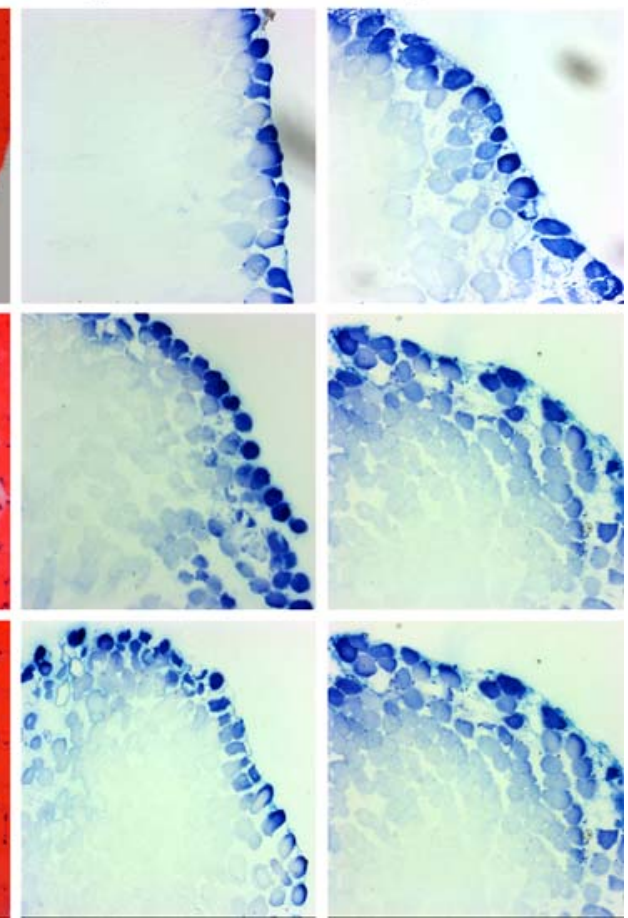

Table 1 Serum CK levels from running mouse cohort

\begin{tabular}{llllll}
\hline Mouse ID & Genotype & CK U/I & Mouse ID & Genotype & CK U/I \\
\hline 0 & $+/+$ & 188 & 0 & $-/-$ & 741 \\
1 & $+/+$ & 447 & 1 & $-/-$ & 1161 \\
3 & $+/+$ & 704 & 3 & $-/-$ & 145 \\
4 & $+/+$ & 410 & 4 & $-/-$ & 1771 \\
10 & $+/+$ & 184 & 10 & $-/-$ & 1545 \\
30 & $+/+$ & 243 & 30 & $-/-$ & 616 \\
+ I+ Average & 362.6 & \multicolumn{7}{c}{- Average } & 996.5 \\
\hline
\end{tabular}

synemin has also been shown to act as a binding partner for desmin and $\alpha$-dystrobrevin, and the spatial organization of synemin exhibits a number of parallels to that of syncoilin (Bhosle et al. 2006). As such, it has the potential to act in a compensatory role in a sync-/- system.

Our Western blot analysis for both $\mathrm{H}$ and $\mathrm{M}$ variants (analogous to $\alpha$ and $\beta$ variants in humans) of synemin showed no difference in net protein levels between sync+/ + and sync-/- mice, indicating that a lack of overt phenotype in the sync-/- mouse is not due to compensation by synemin. It is possible, however, that the presence of synemin in what appears to be a similar role in muscle offsets or masks the deleterious effects of a loss of syncoilin, such that under normal laboratory cage or mild exercise conditions, syncoilin exhibits a degree of functional redundancy.

In summary, our investigations show that we have generated a functional example of a syncoilin knockout mouse model. Syncoilin-null mice do not display an overt phenotype. An absence of syncoilin does not (in the proteins investigated in this study) induce a compensatory protein upregulation in skeletal muscle or the disruption of protein localization in isolated fibers. No difference in muscle regeneration following cardiotoxin-induced necrosis was observed, and an absence of syncoilin did not affect voluntary or enforced exercise capacity.

The mechanical analysis of isolated muscle revealed a significant alteration in contractile properties, indicating that syncoilin may play a role in the lateral transmission of force across contractile tissues. Most interestingly, we have shown that under extended exercise conditions, an absence of syncoilin predisposes muscle to increased damage. Given the findings in this and other studies, it is possible that the role of syncoilin is analogous to that of "a single spoke in a wheel," in that its structural role is not appreciated (nor its absence noted) until significant levels of mechanical stress are applied to the syncoilin-deficient system.

Acknowledgments This work was supported by the Muscular Dystrophy Association USA and Association Française Contre les Myopathies. The authors are grateful to Dr. S. Peirson for providing valuable assistance with the monitored mouse running study, and to D. Jelfs for assistance with animal husbandry.

Open Access This article is distributed under the terms of the Creative Commons Attribution Noncommercial License which permits any noncommercial use, distribution, and reproduction in any medium, provided the original author(s) and source are credited. 


\section{References}

Armand AS, Launay T, Gaspera BD, Charbonnier F, Galllien CL et al (2003) Effects of eccentric treadmill running on mouse soleus: degeneration/regeneration studies with Myf-5 and MyoD probes. Acta Physiol Scand 179:75-84

Bellin RM, Sernett SW, Becker B, Ip W, Huiatt TW et al (1999) Molecular characteristics and interactions of the intermediate filament protein synemin. Interactions with alpha-actinin may anchor synemin-containing heterofilaments. J Biol Chem 274: 29493-29499

Bellin RM, Huiatt TW, Critchley DR, Robson RM (2001) Synemin may function to directly link muscle intermediate filaments to both myofibrillar z-lines and costameres. J Biol Chem 34:3233032337

Benson MA, Newey SE, Martin-Rendon E, Hawkes R, Blake DJ (2001) Dysbindin, a novel coiled-coil-containing protein that interacts with the dystrobrevins in muscle and brain. J Biol Chem 276:24232-24241

Bhosle RC, Michele DE, Campbell KP, Li Z, Robson RM (2006) Interactions of intermediate filament protein synemin with dystrophin and utrophin. Biochem Biophys Res Commun 346: 768-777

Blake DJ, Nawrotzki R, Loh NY, Górecki DC, Davies KE (1998) Beta-dystrobrevin, a member of the dystrophin-related protein family. Proc Natl Acad Sci USA 95:241-246

Blake DJ, Weir A, Newey SE, Davies KE (2002) Function and genetics of dystrophin and dystrophin-related proteins in muscle. Physiol Rev 82:291-231

Brown LD, Schneider MF (2002) Delayed dedifferentiation and retention of properties in dissociated adult skeletal muscle fibers in vitro. In Vitro Cell Dev Biol Anim 38:411-422

Brown SC, Torelli S, Ugo I, De Biasia F, Howman EV et al (2005) Syncoilin upregulation in muscle of patients with neuromuscular disease. Muscle Nerve 32:715-725

Capetanaki Y, Bloch RJ, Kouloumenta A, Mavroidis M, Psarras S (2007) Muscle intermediate filaments and their links to membranes and membranous organelles. Exp Cell Res 313:2063-2076

Costa ML, Escaleria R, Cataldo A, Olivera F, Mermelstein CS (2004) Desmin: molecular interactions and putative functions of the muscle intermediate filament protein. Braz J Med Biol Res 37:1819-1830

Davies KE, Nowak KJ (2006) Molecular mechanisms of muscular dystrophies: old and new players. Nat Rev Mol Cell Biol 7:762773

Haubold KW, Allen DL, Capetanaki Y, Leinwand LA (2003) Loss of desmin leads to impaired voluntary wheel running and treadmill exercise performance. J Appl Physiol 95:1617-1622

Jin H, Tan S, Hermanowski J, Böhm S, Pacheco S et al (2007) The dystrolein, dystrophin and dystrobrevin superfamily: new paralogues and old isoforms. BMC Genomics 8:19

Jones KJ, Compton AG, Yang N, Mills MA, Peters MF et al (2003) Deficiency of the syntrophins and $\alpha$-dystrobrevin in patients with inherited myopathy. Neuromuscul Disord 13:456-467

Koo JH, Smiley MA, Lovering RM, Margolis FL (2007) Bex1 knockout mice show altered skeletal muscle regeneration. Biochem Biophys Res Commun 363:405-410
Kreplak L, Herrmann H, Aebi U (2008) Tensile properties of single desmin intermediate filaments. Biophys J 94:2790-2799

Li Z, Colucci-Guyon E, Pinçon-Raymond M, Mericskay M, Pournin $S$ et al (1996) Cardiovascular lesions and skeletal myopathy in mice lacking desmin. Dev Biol 175:362-366

Lieber RL, Shah S, Fridén J (2002) Cytoskeletal disruption after eccentric contraction-induced muscle injury. Clin Orthop Relat Res S90-S99

McCullagh KJ, Edwards B, Poon E, Lovering RM, Paulin D et al (2007) Intermediate filament-like protein syncoilin in normal and myopathic striated muscle. Neuromuscul Disord 17: 970-979

Milner DJ, Weitzer G, Tran D, Bradley A, Capetanaki Y (1996) Disruption of muscle architecture and myocardial degeneration in mice lacking desmin. J Cell Biol 134:1255-1270

Mizuno Y, Guyon JR, Watkins SC, Mizushima K, Sasaoka T et al (2004) Beta-synemin localizes to regions of high stress in human skeletal myofibers. Muscle Nerve 30:337-346

Moens P, Baatsen PH, Maréchal G (1993) Increased susceptibility of EDL muscles from $m d x$ mice to damage induced by contractions with stretch. J Muscle Res Cell Motil 14:446-451

Newey SE, Howman EV, Ponting CP, Benson MA, Nawrotzki R et al (2001) Syncoilin, a novel member of the intermediate filament superfamily that interacts with alpha-dystrobrevin in skeletal muscle. J Biol Chem 276:6645-6655

Olive M, Goldfarb L, Moreno D, Laforet E, Daqvadori A et al (2004) Desmin-related myopathy: clinical, electrophysiological, radiological, neuropathological and genetic studies. J Neurol Sci 219:125-137

Parry DAD, Strelkov SV, Burkhard P, Aebi U, Herrmann H (2007) Towards a molecular description of intermediate filament structure and assembly. Exp Cell Res 313:2204-2216

Paulin D, Huet A, Khanamyrian L, Xue Z (2004) Desminopathies in muscle disease. J Pathol 204:418-427

Poon E, Howman EV, Newey SE, Davies KE (2002) Association of syncoilin and desmin: linking intermediate filament proteins to the dystrophin-associated protein complex. J Biol Chem 277: 3433-2439

Rees MLJ, Lien CF, Górecki DC (2007) Dystrobrevins in muscle and non-muscle tissues. Neuromuscul Disord 17:123-134

Shi X, Garry DJ (2006) Muscle stem cells in development, regeneration, and disease. Genes Dev 20:1692-1708

Sun N, Critchley DR, Paulin D, Li Z, Robson RM (2008) Human $\alpha$ synemin interacts directly with vinculin and metavinculin. Biolchem J 409:657-667

Vilquin JT, Brussee V, Asselin I, Kinoshita I, Gingras M et al (1998) Evidence of $m d x$ mouse skeletal muscle fragility in vivo by eccentric running exercise. Muscle Nerve 21:567-576

Woods CE, Novo D, DiFranco M, Vergara JL (2004) The action potential-evoked sarcoplasmic reticulum calcium release is impaired in $m d x$ mouse muscle fibres. J Physiol 557:59-75

Zhang J, Bang ML, Gokhin DS, Lu Y, Cui L et al (2008) Syncoilin is required for generating maximum isometric stress in skeletal muscle but dispensable for muscle architecture. Am J Physiol Cell Physiol 294:C1175-C1182 